\title{
Assessment of phenomenological models for viscosity of liquids based on nonequilibrium atomistic simulations of copper
}

\author{
Peng Xu, Tahir Cagin, ${ }^{\text {a) }}$ and William A. Goddard III ${ }^{\text {b) }}$ \\ Materials and Process Simulation Center (I39-74), California Institute of Technology, \\ 1200 East California Boulevard, Pasadena, California 91125
}

(Received 23 July 2004; accepted 2 February 2005; published online 13 September 2005)

\begin{abstract}
The shear viscosity of liquid copper is studied using nonequilibrium molecular-dynamics simulations under planar shear flow conditions. We examined variation of viscosity as function of shear rate at a range of pressures (ca. $0-40 \mathrm{GPa}$ ). We analyzed these results using eight different phenomenological models and find that the observed non-Newtonian behavior is best described by the Powell-Eyring (PE) model: $\eta(\dot{\gamma})=\left(\eta_{0}-\eta_{\infty}\right) \sinh ^{-1}(\tau \dot{\gamma}) /(\tau \dot{\gamma})+\eta_{\infty}$, where $\dot{\gamma}$ is the shear rate. Here $\eta_{0}$ (the zero-shear-rate viscosity) extracted from the PE fit is in excellent agreement with available experimental data. The relaxation time $\tau$ from the PE fit describes the shear response to an applied stress. This provides the framework for interpreting the shear flow phenomena in complex systems, such as liquid metal and amorphous metal alloys. (C) 2005 American Institute of Physics.
\end{abstract}

[DOI: $10.1063 / 1.1881052]$

\section{INTRODUCTION}

The transport properties such as viscosity and associated relaxation time are critical for understanding dynamic behavior of liquids and supercooled liquids. These properties are important in applications in the biology, pharmaceutical and food industries, and to all kinds of materials processing. Unfortunately, it is difficult to obtain experimental data on viscosity adequate for optimizing the performance of processing technology, and there is little fundamental understanding of how these properties depend on microscopic details and the atomic composition of materials under consideration. Several phenomenological approaches (empirical and microscopic) have been developed to relate the complex rheological behaviors of fluids ${ }^{1}$ to the limited experimental data. However, most empirical models have limitations ${ }^{1}$ and do not provide us the fundamental understanding of liquids at the microscopic (atomistic) level. Over the years, various theories have also been developed to provide the microscopic aspects of this problem including Eyring's significant structure theory, ${ }^{2,3}$ Stillinger's energy landscape theory, ${ }^{4}$ and AdamGibbs entropy theory ${ }^{5}$ as well as free volume theory introduced by Turnbull, Cohen, and others. ${ }^{6}$ However, the gap between today's liquid theories and experiments (or empirical models) remains large with the general understanding of liquid phase lagging far behind that of the gas or crystalline phases due to its structural and dynamic complexities. With the rapid increase of computational resources and improvement in computational methods and approaches, simulations with atomistic detail poised to bridge the link between theories and experiments. On one hand, one can simulate model systems for which experimental data exist to reproduce the

\footnotetext{
${ }^{a)}$ Permanent address: Department of Chemical Engineering, Texas A\&M University, College Station, TX 77845-3122.

${ }^{b)}$ To whom correspondence should be addressed. Electronic mail: wag@wag.caltech.edu
}

experimental values or use simulations to calculate new data more easily; on the other hand, one can also deal with many experimentally unpractical cases to test the theories and explore the underlying physics. In this paper, we will present our efforts in this respect by focusing on a relatively simple case - liquid metal (using liquid copper as an example), we study the shear viscosity using nonequilibrium molecular dynamics as a function of shear rate and assess the applicability of various phenomenological models describing viscositystrain rate relationships.

In the following section we will describe the model system, parameters used to model the interactions in liquid copper, and simulation details. In Sec. III we will describe both empirical and microscopic models of viscosity-strain rate relationships and assess their applicability range and validity. In Sec. IV we will give an account and discussion of our simulation results conducted at various densities and strain rates.

\section{SIMULATION DETAILS}

In our simulations we used liquid copper models at $T$ $=2000 \mathrm{~K}$ at various densities. We first constructed random structures at the selected densities, this is then followed by a minimization procedure to eliminate the overlaps, and in the third stage we have used molecular-dynamics simulations to equilibrate the model systems through gradual heating and aging at $T=2000 \mathrm{~K}$. In the following we will give a brief description of the interaction potentials and parameters and nonequilibrium molecular-dynamics method used in this simulation study.

\section{A. Interaction potential and parameters}

In our simulations, we used a many-body force field (FF) of Sutton-Chen (SC) type. ${ }^{7}$ The total potential energy of the metal in SC type is described as 
TABLE I. Sutton-Chen $(Q$-SC) force fields parameters used for copper in this work.

\begin{tabular}{cccccc}
\hline \hline & $D(\mathrm{meV})$ & $c$ & $m$ & $n$ & $\alpha(\AA)$ \\
\hline $\mathrm{Cu}$ & 5.7921 & 84.843 & 5 & 10 & 3.6030 \\
\hline \hline
\end{tabular}

$$
U_{\mathrm{tot}}=\sum_{i} U_{i}=\sum_{i} D\left[\sum_{j \neq i} \frac{1}{2} V\left(r_{i j}\right)-c \rho_{i}{ }^{1 / 2}\right]
$$

where $r_{i j}$ is the distance between atoms $i$ and $j, V\left(r_{i j}\right)$ is a pairwise potential,

$$
V\left(r_{i j}\right)=\left(\frac{\alpha}{r_{i j}}\right)^{n},
$$

accounting for the short-range repulsion between the atoms core $i$ and $j$, and $\rho_{i}$ is the local density associated with atom $i$ defined as

$$
\rho_{i}=\sum_{j \neq i} \phi\left(r_{i j}\right)=\sum_{j \neq i}\left(\frac{\alpha}{r_{i j}}\right)^{m} .
$$

The sums over $i-j$ pairs in Eqs. (1)-(3) are performed for all $r_{i j}<2 a$, i.e., the cutoff distance of twice the lattice parameter is used. In the above equations, $D$ sets the overall energy scale, while $c$ is a dimensionless parameter scaling the attractive term, and $\alpha$ is a length parameter leading to a dimensionless form for $V$ and $\rho$.

The optimized parameters used in this simulation are given in Table I. These parameters were obtained by fitting the density, cohesive energy, elastic constant, and the phonon-dispersion curves. They lead to accurate values for surface energies, vacancy formation energy, and stacking fault energy for crystal phases. ${ }^{8}$ We refer to this modified set of SC parameters as the $Q$-SC force fields since the fitting has included quantum effects, i.e., the zero-point energy and pressure. We also studied the liquid structure and solidliquid phase transition of copper using this force field ${ }^{9}$ to make sure that the potential is adequate for our viscosity study.

Our models of equilibrated liquid $\mathrm{Cu}$ contained 1000 atoms in a cubic cell with three-dimensional periodic boundary conditions. The densities of these model systems were $6.753,7.088,7.633,8.027,8.672$, and $9.909 \mathrm{~g} / \mathrm{cm}^{3}$. We have conducted 20-ps long constant temperature equilibrium molecular-dynamics simulations to determine the corresponding pressures to these densities. They are $-0.62,1.11$, $5.04,8.80,11.18$, and $42.29 \mathrm{GPa}$, respectively.

\section{B. NEMD method}

To study the viscosity as a function of strain rate we have employed the nonequilibrium molecular-dynamics (NEMD) method, which is carried out for a system subject to a planar Couette flow (pure shear), with fixed density and temperature (temperature is fixed via a Gaussian thermostat). We have considered that the applied shear is in the $x y$ plane and changes linearly in the $y$ direction (see Fig. 1).

The described planar shear flow in the system can be modeled by applying the following isokinetic SLLOD (re-

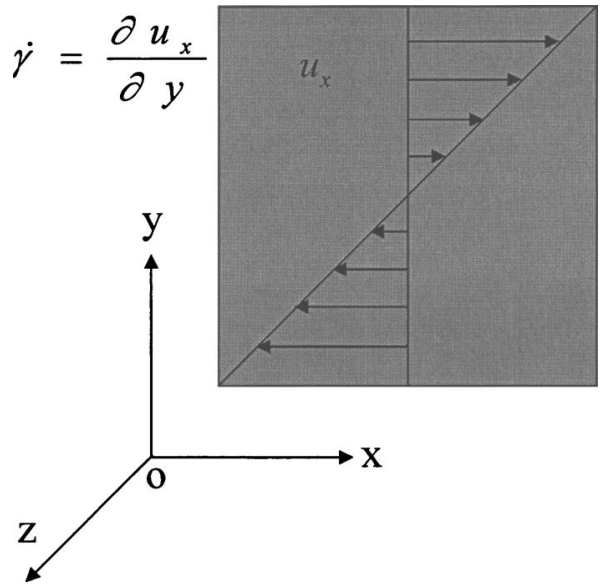

FIG. 1. Schematic representation NEMD simulation geometry and flow velocity profile.

versed spelling of DOLLS, it uses the transpose of the $\boldsymbol{q p}$ DOLLS tensor) ${ }^{10}$ equations of motion for the fluid. ${ }^{10,11}$

$$
\begin{aligned}
& \left\{\begin{array}{l}
\dot{x}_{i}=\frac{P_{x i}}{m_{i}}+\dot{\gamma} y_{i} \\
\dot{y}_{i}=\frac{P_{y i}}{m_{i}} \\
\dot{z}_{i}=\frac{P_{z i}}{m_{i}},
\end{array}\right. \\
& \left\{\begin{array}{l}
\dot{P}_{x i}=F_{x i}-\alpha P_{x i}-\dot{\gamma} P_{y i} \\
\dot{P}_{y i}=F_{y i}-\alpha P_{y i} \\
\dot{P}_{z i}=F_{z i}-\alpha P_{z i},
\end{array}\right.
\end{aligned}
$$

where $\dot{\gamma}$ is the shear rate, or the velocity gradient,

$$
\dot{\gamma}=\frac{\partial u_{x}}{\partial y} .
$$

Here $\alpha$ is the Gaussian thermostat variable, its value is determined at each iteration step by solving the following constraint equation:

$$
\alpha=\frac{\sum_{i} \vec{F}_{i} \cdot \vec{P}_{i}-\dot{\gamma} \sum_{i} P_{x i} P_{y i}}{\sum_{i} \vec{P}_{i} \cdot \vec{P}_{i}}
$$

in order to keep the kinetic-energy constant, i.e., resulting in isokinetic-energy simulation condition. During simulations, we employed shear boundary conditions and used a 1-fs time step for integrating the equations of motion.

The shear viscosity $\eta(\dot{\gamma}, T)$ is defined as the ratio of the shear component of the stress tensor $\sigma_{x y}$ and the applied shear rate $\dot{\gamma}$ in fluid mechanics,

$$
\eta=-\frac{\left\langle\sigma_{x y}\right\rangle}{\dot{\gamma}} .
$$

Here, the relevant component of the stress tensor is given by 


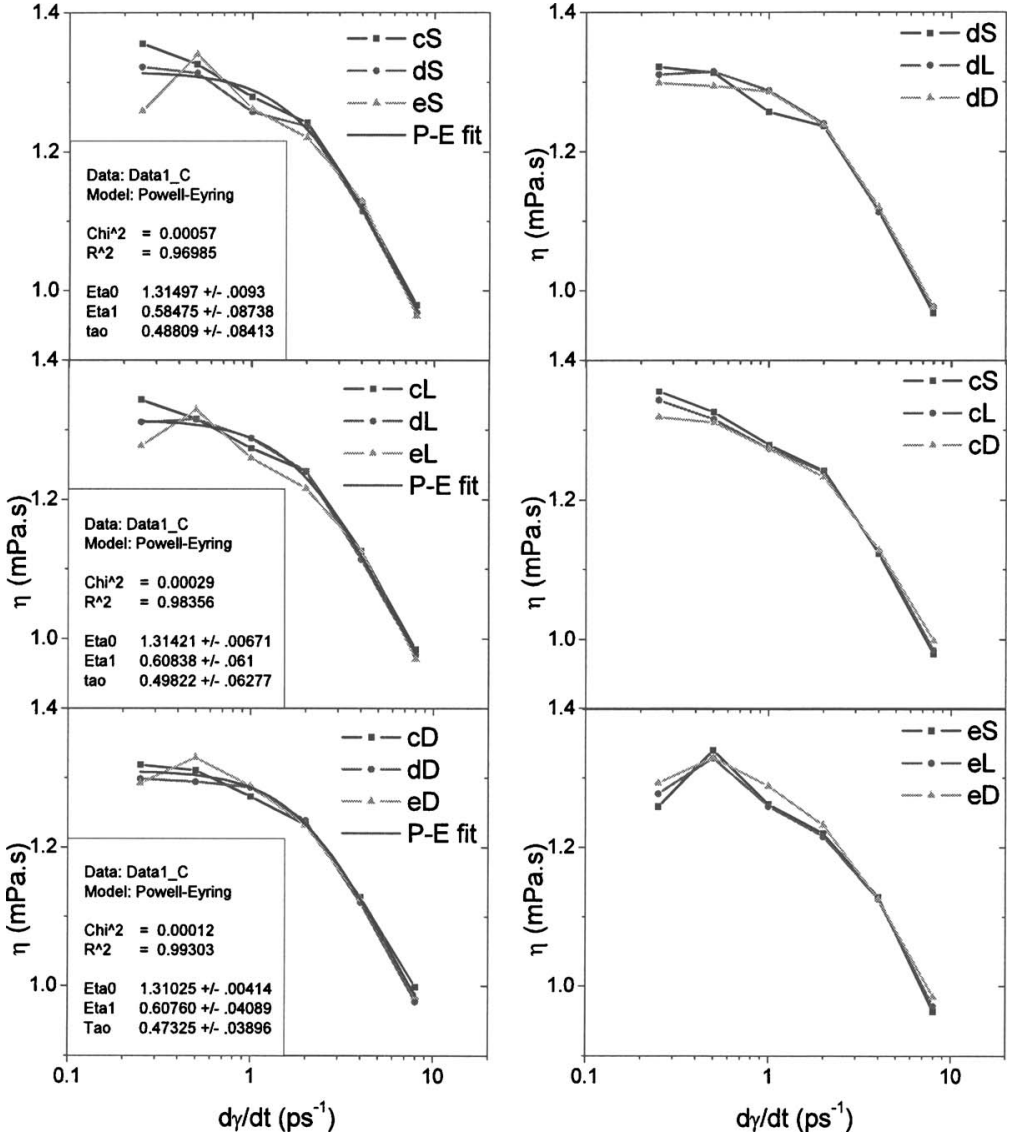

where, $\Omega$ is the volume of the model system and $W_{x y}$ is the relevant component of the virial tensor,

$$
W_{x y}=\frac{1}{2} \sum_{i, j}^{N} r_{x i j} F_{y i j} .
$$

Before collecting data and calculating viscosities using the above equations, we need first to obtain a homogeneous shear field in the simulation models to satisfy the fluid mechanics postulate. Therefore, we started from equilibrated structures from normal MD and then sheared the system for several equilibration cycles before data collection. (A cycle is the time taken to reach from zero to a shear strain value of 1, i.e., 1 ps for shear rate $1-\mathrm{ps}^{-1}$ run and $100 \mathrm{ps}$ for $0.01-\mathrm{ps}^{-1}$ run.) From our tests, the minimum equilibration time varies for each strain rate, and there is remarkable initial structure dependency (Fig. 2). It is obvious that longer equilibration time is needed at low strain rate cases for different initial structures to relax and converge to the same
$S$-shape flow behaviors. It makes sense since there are some intermolecular correlations left from the original structure during the shearing progress especially in low strain rate cases, while much stronger external disturbance in high strain rate cases could break most of the intermolecular correlations and form a homogeneous shear field more easily. According to our tests, we choose 4 cycles as the equilibration length for high strain rate cases $\left(0.25-8 \mathrm{ps}^{-1}\right)$ and $8-10$ cycles for low shear-rate cases $\left(0.1-0.01 \mathrm{ps}^{-1}\right)$. The data collection lengths varied from 40 cycles $\left(0.25-8 \mathrm{ps}^{-1}\right)$ to 10 cycles for $0.01 \mathrm{ps}^{-1}$, making sure acceptable convergence in the average shear stress (less than $1 \%$ variation in the running average) achieved.

\section{PHENOMENOLOGICAL MODELS OF VISCOSITY- STRAIN RATE RELATIONSHIPS AND DISCUSSIONS}

\section{A. Shear-rate dependence of the viscosity of a typical pseudoplastic liquid}

At the experimentally accessible low strain rates, liquid metals and alloys are considered as good Newtonian fluids,

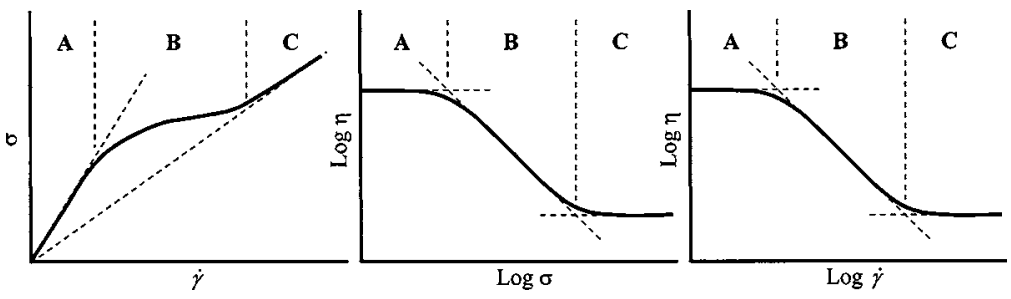

FIG. 3. Qualitative flow behavior of typical pseudoplastic fluids. 
TABLE II. Common empirical models used to describe viscosity of pseudoplastic fluids.

\begin{tabular}{|c|c|c|c|c|c|}
\hline Equation & Model Name & Model Expression & & $\begin{array}{c}\text { Sample Curve } \\
\log \eta \sim \log \sigma\end{array}$ & $\begin{array}{l}\text { Depicted } \\
\text { Stages }\end{array}$ \\
\hline$(11 b)$ & Power Model & $\eta\left(\sigma^{2}\right)=m^{1 / n}\left(\sigma^{2}\right)^{-(1-n) / 2 n}$ & $(n<1)$ & & B \\
\hline$(12)$ & Ellis Model & $\frac{1}{\eta\left(\sigma^{2}\right)}=\frac{1}{\eta_{0}}+m^{-1 / n}\left(\sigma^{2}\right)^{(1-n) / 2 n}$ & $(n<1)$ & & $A$ and $B$ \\
\hline (13) & Sisko Model & $\eta\left(\sigma^{2}\right)=\eta_{\infty}+m^{1 / n}\left(\sigma^{2}\right)^{-(i-n) / 2 n}$ & $(n<1)$ & & $B$ and $C$ \\
\hline (14) & $\begin{array}{l}\text { Reiner- } \\
\text { Philippoff } \\
\text { Model }\end{array}$ & \multicolumn{2}{|l|}{$\eta\left(\sigma^{2}\right)=\eta_{\infty}+\frac{\eta_{0}-\eta_{\infty}}{1+\sigma^{2} / G}$} & & $\begin{array}{l}\mathrm{A}, \mathrm{B} \text { and } \mathrm{C} \\
\text { but not } \\
\text { usually }\end{array}$ \\
\hline (15) & $\begin{array}{c}\text { Reiner's } \\
\text { structural } \\
\text { stability model }\end{array}$ & $\frac{1}{\eta\left(\sigma^{2}\right)}=\frac{1}{\eta_{\infty}}-\left(\frac{1}{\eta_{\infty}}-\frac{1}{\eta_{0}}\right) e$ & $-\sigma^{2} / x^{2}$ & & $\begin{array}{c}\text { quantitatively } \\
\text { exact }\end{array}$ \\
\hline
\end{tabular}

but from our current and previous simulations, ${ }^{11}$ they show a rather "pseudoplastic" fluid behavior, which means that they undergo "shear thinning" at sufficiently high strain rates, i.e., its viscosity decreases as the strain rate rises. Apparently, this difference comes from the disparity in time scales involved in simulations and low shear-rate experiments: The characteristic time scales of simulations range from picoseconds to nanoseconds, hence the shear rates explored by NEMD methods are at least on the order of $10^{10} \mathrm{~s}^{-1}$ and mostly one to two orders of magnitude larger. Obviously these rates are much larger than the experimental rates. The rheological be- havior of a typical pseudoplastic fluid is shown in Fig. 3. ${ }^{1}$ From the $S$-shaped curve, one can identify three stages of the typical flow behavior of a pseudoplastic fluid: $A$, pseudoNewtonian at nearly zero shear rates; $B$, shear-thinning period; $C$, pseudo-Newtonian at nearly infinite shear rates. In experiments, the shear rates are normally within the stage $A$, and the liquid metals behave like a Newtonian fluid, but in simulations, our shear rates cover all of the three stages. To compare with experiments and understand the shear-thinning behavior of liquid metal, it is necessary to figure out the shear-rate dependence of viscosity first.

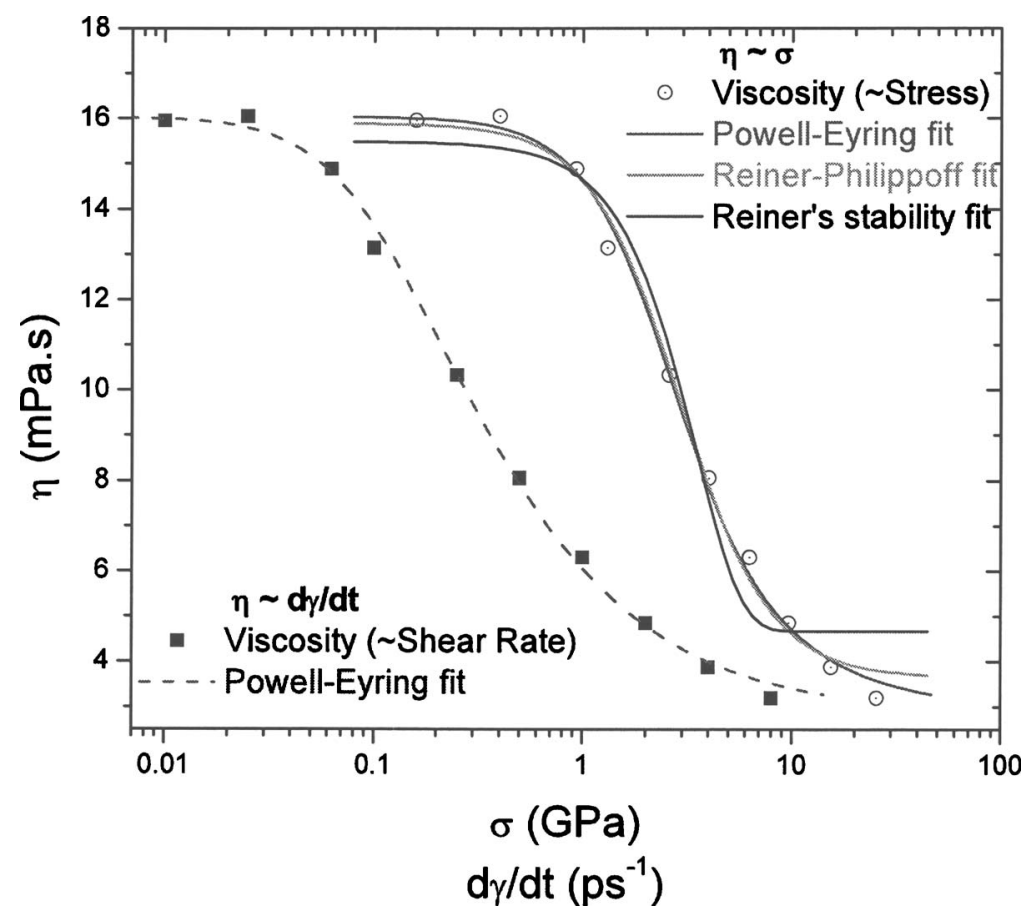

FIG. 4. Comparison between Reiner-Philippoff model [Eq. (14)], Reiner's structural stability model [Eq. (15)], and Powell-Eyring model [Eq. (18)] in fitting shear-rate dependence of viscosities of $\mathrm{Cu}$ at $T=2000 \mathrm{~K}$, with density fixed at $d=9.909 \mathrm{~g} / \mathrm{cm}^{3}$, corresponding to an equilibrium pressure, $P=42.294 \mathrm{GPa}$. 
TABLE III. The microscopic models of pseudoplastic fluids considered in this work.

\begin{tabular}{|c|c|c|c|c|c|}
\hline Equation & Model name & Model expression & & $\frac{d \eta}{d \dot{\gamma}}$ & $\left.\underline{\eta}\right|_{j=0}$ \\
\hline (16) & Cross model & $\eta(\dot{\gamma})=\eta_{\infty}+\frac{\eta_{0}-\eta_{\infty}}{1+(\tau \dot{\gamma})^{1-n}} \quad(n<1)$ & & $\left\{\begin{array}{l}-\infty \\
-\tau\left(\eta_{0}-\eta_{\infty}\right) \\
0\end{array}\right.$ & $\begin{array}{l}(0<n<1) \\
(n=0) \\
(n<0)\end{array}$ \\
\hline (17) & Carreau model & $\eta(\dot{\gamma})=\eta_{\infty}+\left(\eta_{0}-\eta_{\infty}\right)\left[1+(\tau \dot{\gamma})^{2}\right]^{(n-1) / 2}$ & $(n<1)$ & & 0 \\
\hline (18) & Powell-Eyring model & $\eta(\dot{\gamma})=\left(\eta_{0}-\eta_{\infty}\right) \frac{\sinh ^{-1}(\tau \dot{\gamma})}{\tau \dot{\gamma}}+\eta_{\infty}$ & & & 0 \\
\hline (19) & Johnson model & $\eta(\dot{\gamma})=\eta_{\infty} \exp \left(\frac{1}{\frac{1}{\ln \eta_{0}-\ln \eta_{\infty}}+\tau \dot{\gamma}}\right)$ & & $-\tau \eta_{0}(1$ & $\ln \left(\frac{\eta_{0}}{\eta_{\infty}}\right)^{2}$ \\
\hline
\end{tabular}

\section{B. Empirical models of viscosity-strain rate relationship}

Empirical models generally use stress as their independent variable to fit the $S$-shaped "pseudoplastic" flow curves since it is easy to control experimentally. The most popular empirical models are summarized in Table II. Among them, the simplest one is the power-law model of Ostwald-de Waele-Nutting [Eq. (11b)].112 It also has a simpler form using shear rate as independent variable

$$
\sigma=m(\dot{\gamma})^{n}, \quad \eta=m(\dot{\gamma})^{n-1} .
$$

When $n<1$, the power-law model fits the shear-thinning stage $B$ well, but it cannot represent the stages $A$ and $C$. It diverges when the stress or shear rate approaches zero. Hence, it is impossible to use it to extrapolate to the zero- shear-rate viscosity. Extensions of the power-law model by Ellis [Eq. (12) $]^{1,15}$ and by Sisko [Eq. (13) $]^{13}$ represent only two of the three stages well, respectively (the stages $A-B$ and $B-C)$, but not all three. Even the models which can be used to fit the $S$-shaped curve of Fig. 3 like the Reiner-Philippoff model [Eq. (14)] $]^{14}$ and Reiner's structural stability model [Eq. (15) $]^{15}$ are not usually quantitatively accurate. We compare in Fig. 4 these two models [Eqs. (14) and (15)] with the microscopic Powell-Eyring model [Eq. (18)], where we see that they lead to a poorer fit to our data than the PowellEyring model. In addition, stress as the independent variable in these methods is not as easy to control in simulations as strain rate (the independent variable of Powell-Eyring model). Therefore, we prefer the Powell-Eyring model to these empirical models for fitting to our data.

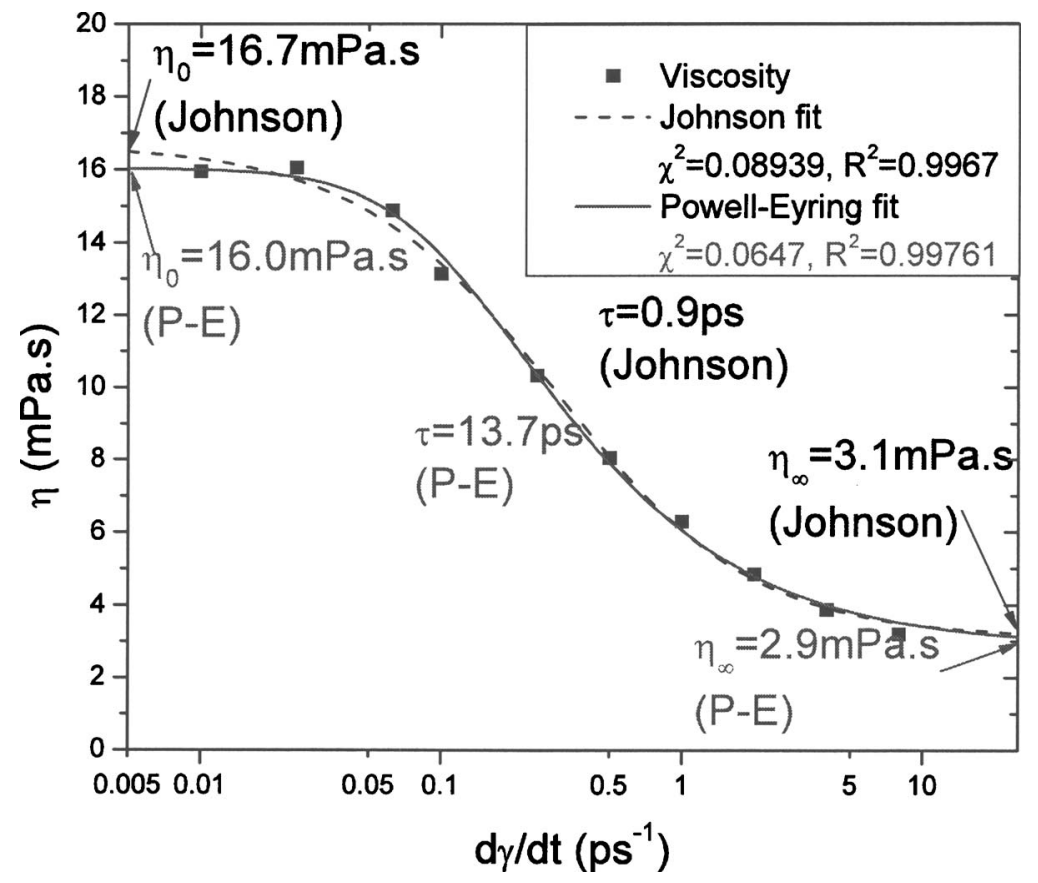

FIG. 5. Comparison between Johnson model [Eq. (19)] and Powell-Eyring model [Eq. (18)] in fitting shear-rate dependence of viscosities of $\mathrm{Cu}$ at $T=2000 \mathrm{~K}$, with density fixed at $d=9.909 \mathrm{~g} / \mathrm{cm}^{3}$, corresponding to an equilibrium pressure, $P=42.294 \mathrm{GPa}$. 


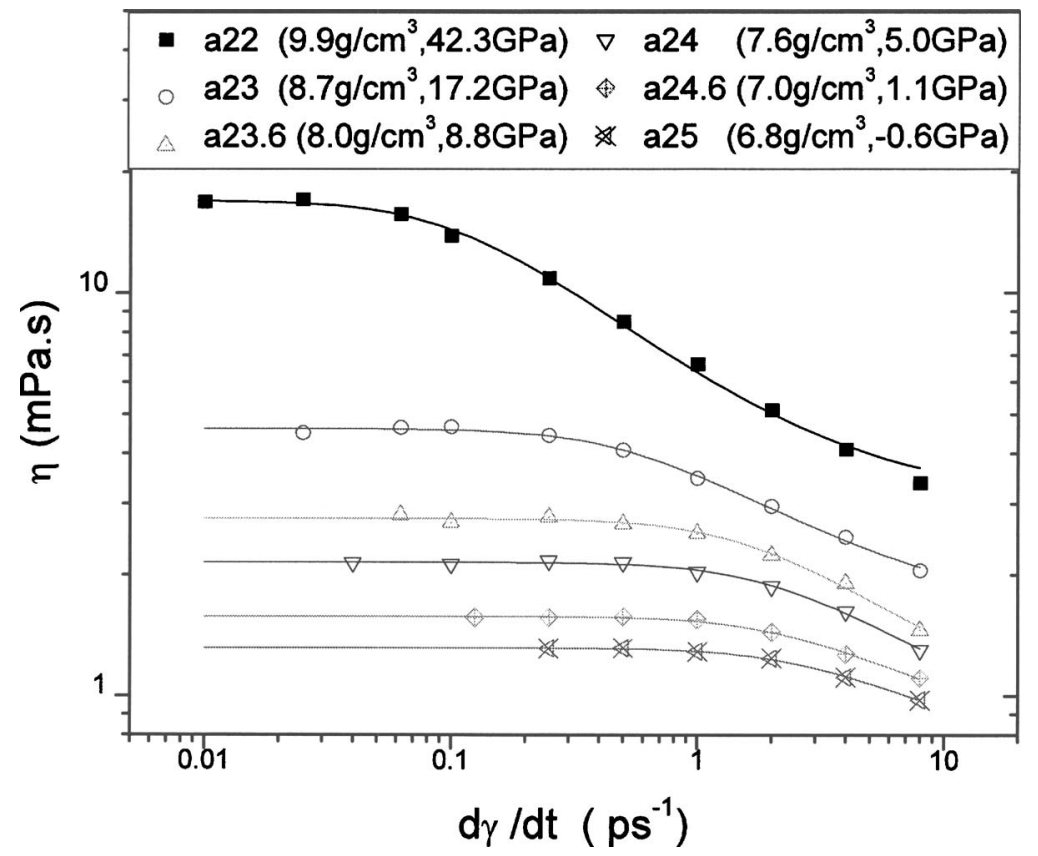

FIG. 6. Viscosities of liquid $\mathrm{Cu}$ under different pressures and constant $T=2000 \mathrm{~K}$.

\section{Microscopic models of viscosity-strain rate based on microscopic theories}

Starting from different assumptions on the physical processes involved in the flow phenomena in liquids, the microscopic theories usually lead to models with shear rate as the independent variable. There are some microscopic models which captured the well-known $S$-shaped flow curve (Fig. 2), such as Cross model [Eq. (16)], ${ }^{16}$ Carreau model [Eq. (17)], ${ }^{17,18}$ Powell-Eyring model [Eq. (18)],,$^{3,19}$ as well as Johnson model $[\mathrm{Eq} .(19)]^{20}$ recently developed from free volume theory. Their functional forms and characteristics are summarized in Table III. According to the $S$-shaped flow curve of a typical pseudoplastic fluid as Fig. 3, the two asymptotic limits for viscosities: $\eta(\dot{\gamma}=0)=\eta_{0}$ and $\eta(\dot{\gamma} \rightarrow \infty)$ $=\eta_{\infty}$ should at least be included in the model equation as the necessary parameters. Another parameter $\tau$ as the corresponding characteristic relaxation time should also be introduced. This makes $(\tau \dot{\gamma})$ a dimensionaless variable in the formulations. The increase in $\tau$ will only shift the curve rightwards without any other effects on these models. Thus, all shear rate related terms could be expressed in terms of the product $(\tau \dot{\gamma})$, i.e., $\eta\left(\eta_{0}, \eta_{\infty}, \tau, \ldots, \dot{\gamma}\right)=\eta\left(\eta_{0}, \eta_{\infty}, \ldots, \tau \dot{\gamma}\right)$. The models mentioned above, all satisfy these conditions and previous studies ${ }^{2,18,19,21}$ have shown their suitability in fitting the data for different material systems.

Here, we will focus on two particular functional features to distinguish them and choose the best to fit our data. First, the Cross and Carreau models have the same power-law asymptotic behavior in the shear-thinning range: $\eta(\dot{\gamma}) \sim\left(\eta_{0}\right.$ $\left.-\eta_{\infty}\right)(\tau \dot{\gamma})^{n-1}$, where $(n-1)$ is the slope of the power-law re-

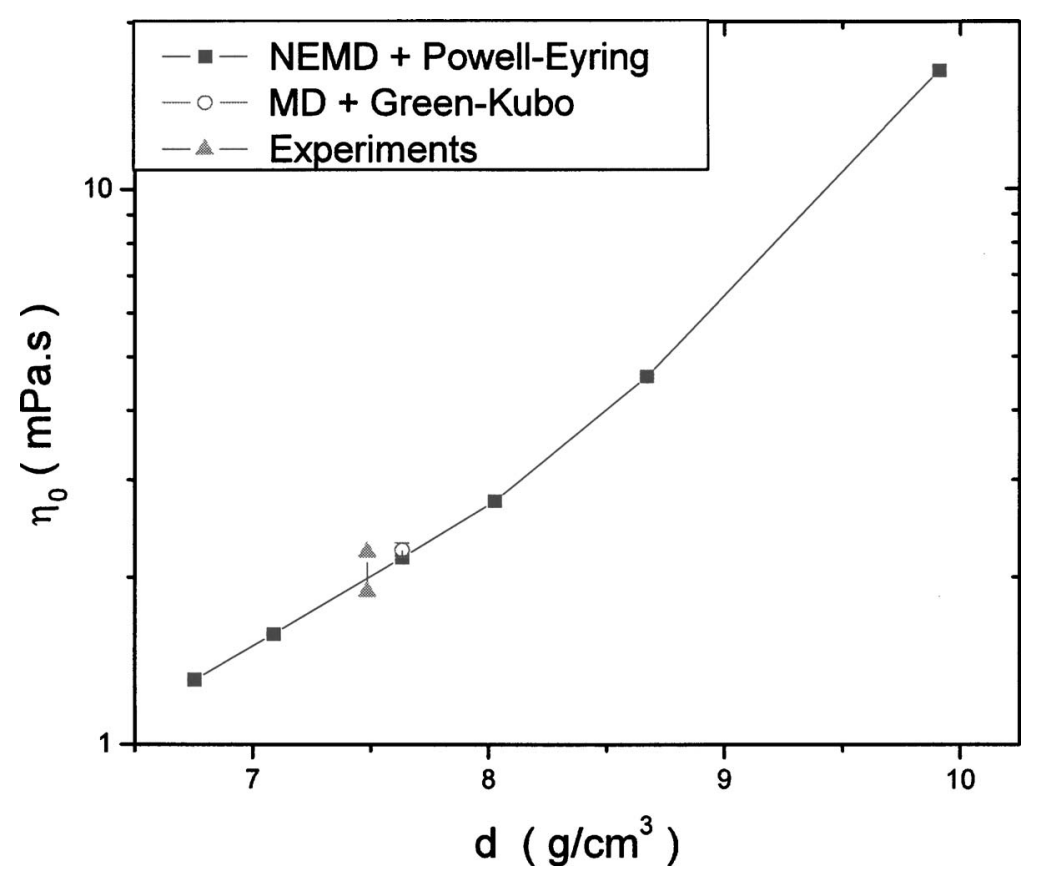

FIG. 7. Powell-Eyring fitted zero-shear-rate viscosities of $\mathrm{Cu}(T=2000 \mathrm{~K})$ compared with experimental data (density from Ref. 22 and viscosities from Refs. 22,23) as well as values calculated from normal MD simulations and Green-Kubo equation. 
gion of the $\log \eta \sim \log \dot{\gamma}$ plot. Therefore, their shear-thinning range $\left(\sim\left(\log \eta_{0}-\log \eta_{\infty}\right) /|n-1|\right)$ is essentially controlled by a fourth parameter (the exponent parameter, $n$ ) as well as $\eta_{0}$ and $\eta_{\infty}$. However, in the other three-parameter models (Powell-Eyring and Johnson) the shear-thinning ranges are always around two orders of magnitude independent of parameters. While the very large shear-thinning variations among polymer solutions may require such flexible models as Cross and Carreau equations, we found that the shearthinning ranges in liquid metal and alloy systems always cover two orders of magnitude in $\log \eta \sim \log \dot{\gamma}$ or $\eta \sim \log \dot{\gamma}$ plots independent of state variables (temperature, pressure, etc.) and system complexities. For these cases, the threeparameter Powell-Eyring and Johnson models are more concise and suitable with all three parameters having real physical significance. Moreover, the similar quality fits (Fig. 4) resulting from both equations with different theoretical explanations imply that we might have already captured the character of the flow behavior of liquid metal system, but the underlying physics may need further study. Another important feature that the model should have in order to describe Newtonian fluid in the low strain rate region is that the derivative of viscosity with respect to strain rate is zero at zero strain rate value, i.e., $\eta(\dot{\gamma})=\eta_{0}+o\left(\dot{\gamma}^{2}\right)$ in the vicinity of $\dot{\gamma}$ $=0$. From Table III, we see that the Carreau and PowellEyring models satisfy this requirement and the Cross model satisfies it only under certain conditions $(n<0)$ while the Johnson model does not. Figure 5 shows the result of fitting one set of our simulation results to these two models, and we see that the Powell-Eyring model represents the data better than Johnson's expression in the low shear-rate region. Therefore, the zero-shear-rate viscosity we obtained from the Powell-Eyring model is more accurate than the one Johnson model gave. To compare with the experimental pseudoNewtonian viscosity at very low shear rate, we use the Powell-Eyring model to deduce the zero-shear-rate viscosity in the following simulations.
TABLE IV. Calculated Powell-Eyring model parameters for liquid $\mathrm{Cu}$ at $T=2000 \mathrm{~K}$.

\begin{tabular}{lccccc}
\hline \hline $\begin{array}{c}\text { Box size } \\
(\AA)\end{array}$ & $\begin{array}{c}P \\
(\mathrm{GPa})\end{array}$ & $\begin{array}{c}d \\
\left(\mathrm{~g} / \mathrm{cm}^{3}\right)\end{array}$ & $\begin{array}{c}\eta_{0} \\
(\mathrm{mPa} \mathrm{s})\end{array}$ & $\begin{array}{c}\eta_{\infty} \\
(\mathrm{mPa} \mathrm{s})\end{array}$ & $\begin{array}{c}\tau \\
(\mathrm{ps})\end{array}$ \\
\hline 22 & 42.294 & 9.909 & 16.04 & 2.874 & 13.73 \\
23 & 17.179 & 8.672 & 4.605 & 1.546 & 2.624 \\
23.6 & 8.800 & 8.027 & 2.759 & 0.722 & 0.890 \\
24 & 5.036 & 7.633 & 2.151 & 0.637 & 0.667 \\
24.6 & 1.112 & 7.088 & 1.580 & 0.675 & 0.600 \\
25 & -0.623 & 6.753 & 1.318 & 0.592 & 0.493 \\
\hline \hline
\end{tabular}

\section{RESULTS AND DISCUSSIONS}

Figure 6 shows the viscosity-shear-rate curves of copper at the same temperature $T=2000 \mathrm{~K}$ but at different densities (pressures). All data are well represented by the PowellEyring equation and the calculated parameters for the fit are listed in Table IV.

Our calculated zero-shear-rate viscosities are compared with the experimental data (only available under ambient pressure) and the values calculated from normal MD simulation with Green-Kubo equation (only one case at fixed density of $d=7.633 \mathrm{~g} / \mathrm{cm}^{3}$ is calculated) in the viscositydensity plot (Fig. 7), and the agreement is very good. Obviously, the transport properties such as viscosity and relaxation time (Figs. 7 and 8) are strongly dependent on density at fixed temperature. In a previous study, Qi et al. ${ }^{11}$ attributed the temperature dependence of viscosity mainly to the variation of density with temperature (i.e., the increase of free volume). These insights from simulations are in accord with the well-known free volume theory of liquids. ${ }^{6}$ The strong dependence of viscosity on density (or pressure) at elevated pressure is especially relevant for studies of shocked liquids in the context of computational fluid dynamics (CFD) where the viscosity of the fluid is used as an input parameter.

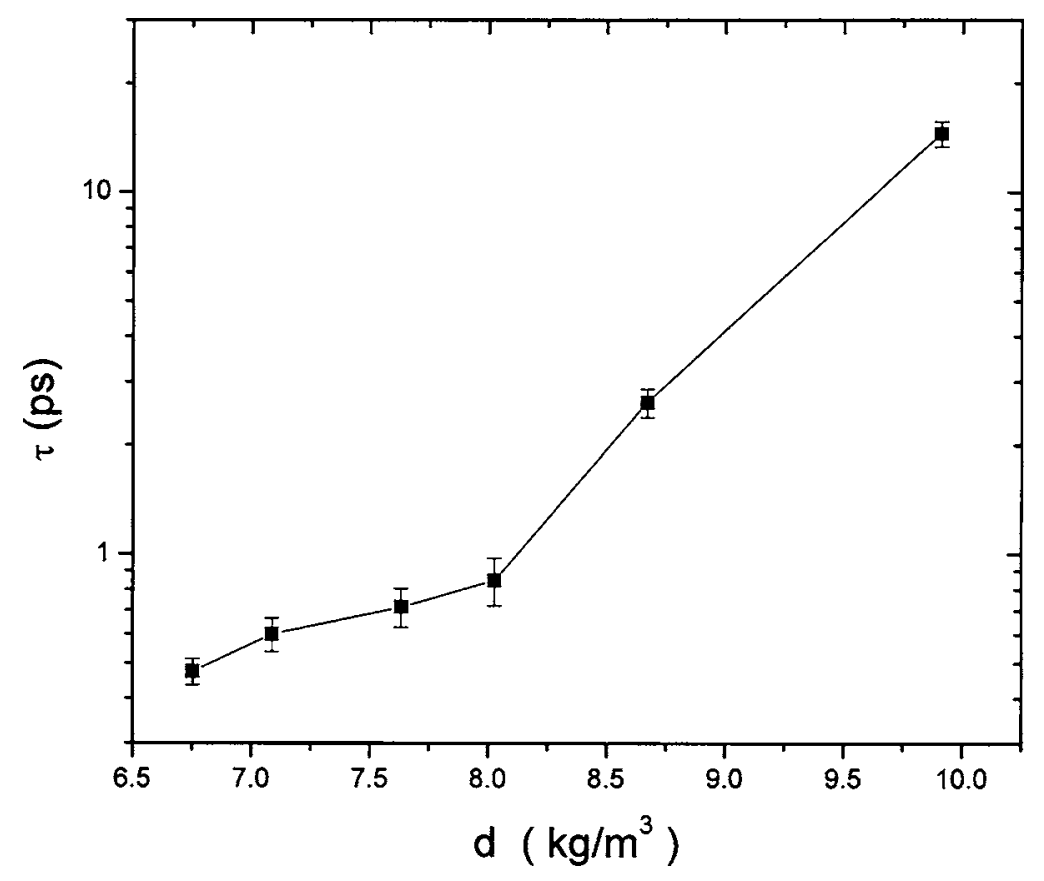

FIG. 8. Powell-Eyring fitted relaxation time of $\mathrm{Cu}(T$ $=2000 \mathrm{~K})$. 


\section{SUMMARY}

In this paper, we study thoroughly the shear-rate dependence of viscosity of liquid copper using NEMD simulations over a range of pressures. We assessed the applicability of various phenomenological models and found that the Powell-Eyring model is the most suitable. This model leads to a zero-shear-rate viscosity of liquid copper that compare well with available experimental data. This analysis leads to a relaxation time that captures the response behavior of the liquid to applied stress. We find a strong dependency of viscosity on density at constant temperature, confirming previous simulation results. This provides the framework for interpreting the shear flow phenomena in complex systems, such as liquid metal alloys and amorphous metal alloys.

\section{ACKNOWLEDGMENTS}

We thank Professor Bill Johnson for many useful discussions. This research was funded by the DARPA ARO-SAM program (Leo Christodoulou and Bill Mullins) and by the NSF-MRSEC (Center for the Science and Engineering Materials, CSEM). The facilities of the Materials and Process Simulation Center are also supported by the Department of Energy in addition to the National Science Foundation, ARO-MURI, MURI-ONR, General Motors, Chevron Texaco, Seiko-Epson, Nissan Corp., and the Beckman Institute.

${ }^{1}$ A. G. Fredrickson, Principles and Applications of Rheology (PrenticeHall, Englewood Cliffs, NJ, 1964).

${ }^{2}$ H. Eyring, D. Henderson, B. J. Stover, and E. M. Eyring, Statistical Mechanics and Dynamics (Wiley, New York, 1964).

${ }^{3}$ F. H. Ree, T. Ree, and H. Eyring, Ind. Eng. Chem. 50, 1036 (1958).

${ }^{4}$ F. H. Stillinger and T. A. Weber, Phys. Rev. A 25, 978 (1982); F. H.
Stillinger, Phys. Rev. E 59, 48 (1999); F. H. Stillinger, Science 267, 1935 (1995).

${ }^{5}$ G. Adam and J. H. Gibbs, J. Chem. Phys. 43, 139 (1965).

${ }^{6}$ D. Turnbull and M. Cohen, J. Chem. Phys. 34, 120 (1961); 52, 3038 (1970); A. Matheson, ibid. 44, 695 (1966); P. Macedo and T. Litovitz, ibid. 42, 245 (1965).

${ }^{7}$ H. Rafiitabar and A. Sutton, Philos. Mag. Lett. 63, 217 (1991); A. Sutton and J. Chen, ibid. 61, 139 (1990).

${ }^{8}$ T. Cagin, Y. Qi, H. Li, Y. Kimura, H. Ikeda, W. L. Johnson, and W. A. Goddard, Bulk Metallic Glasses, MRS Symp. Series, edited by W. L. Johnson, A. Inoue, and C. T. Liu, 554, 43 (1999); Y. Qi, T. Cagin, and W. A. Goddard, Phys. Rev. B 59, 3527 (1999); H. J. Lee, T. Cagin, W. A. Goddard, and W. L. Johnson, J. Metastable Nanocryst. Mater. 15-16, 181 (2003); H. J. Lee, T. Cagin, W. A. Goddard, and W. L. Johnson, J. Chem. Phys. 119, 9558 (2003).

${ }^{9}$ See EPAPS Document No. E-JCPSA6-122-509516 for detailed studies about the solid and liquid phases of copper using $Q$-SC force field. A direct link to this document may be found in the online article's HTML reference section. The document may also be reached via the EPAPS homepage (http://www.aip.org/pubservs/epaps.html) or from ftp.aip.org in the directory /epaps/. See the EPAPS homepage for more information.

${ }^{10}$ D. J. Evans and G. P. Morriss, Statistical Mechanics of Nonequilibrium Liquids (Academic, London, 1990).

${ }^{11}$ Y. Qi, T. Cagin, Y. Kimura, and W. Goddard, J. Comput.-Aided Mater. Des. 8, 233 (2002).

${ }^{12}$ W. Ostwald, Kolloid-Z. 38, 261 (1926).

${ }^{13}$ A. W. Sisko, Ind. Eng. Chem. 50, 1789 (1958).

${ }^{14}$ W. Philippoff, Kolloid-Z. 71, 1 (1935).

${ }^{15}$ M. Reiner, Deformation, Strain and Flow (Interscience, New York, 1960).

${ }^{16}$ M. M. Cross, J. Colloid Sci. 20, 417 (1965).

${ }^{17}$ P. J. Carreau, Trans. Soc. Rheol. 16, 99 (1972).

${ }^{18}$ R. B. Bird, R. C. Armstrong, and O. Hassager, Dynamics of Polymeric Liquids, 2nd ed. (Wiley, New York, 1987).

${ }^{19}$ E. Christiansen, N. Ryan, and W. Stevens, AIChE J. 1, 544 (1965).

${ }^{20}$ W. L. Johnson (personal communications, unpublished).

${ }^{21}$ C. W. Macosko, Rheology Principles, Measurements, and Applications (Wiley, New York, 1993); A. H. P. Skelland, Non-Newtonian Flow and Heat Transfer (Wiley, New York, 1967).

${ }^{22}$ C. J. Smithells, Smithells Metals Reference Book, 8th ed. (Elsevier Butterworth-Heinemann, Burlington, MA, 2004).

${ }^{23}$ M. Hirai, ISIJ Int. 33, 251 (1993). 